\title{
The association between aquaporin-1 expression, microvessel density and the clinicopathological features of hepatocellular carcinoma
}

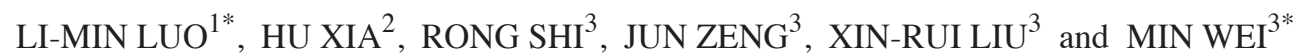 \\ ${ }^{1}$ Centre for Liver Disease, 458th Hospital of People's Liberation Army, Guangzhou, Guangdong 510602; \\ ${ }^{2}$ Department of Respiration Medicine, Zhujiang Hospital, Guangzhou, Guangdong 510282; \\ ${ }^{3}$ Clinical Laboratory, Nanshan Maternity \& Child Healthcare Hospital, Shenzhen, Guangdong 518067, P.R. China
}

Received November 24, 2015; Accepted May 18, 2017

DOI: $10.3892 / \mathrm{ol} .2017 .7106$

\begin{abstract}
The present study aimed to evaluate the effects of aquaporin-1 (AQP1) level and intratumoral microvessel density (IMD) on the clinicopathological features of patients with hepatocellular carcinoma (HCC). The AQP1 expression levels, IMD and AQP1/IMD ratios in patients with HCC were measured using a semi-quantitative immunohistochemical technique. The association between these features and clinicopathological variables were evaluated. The prognostic impact of AQP1 and IMD on overall survival (OS), and 5-year disease-free survival (DFS) of HCC patients was investigated retrospectively. $\mathrm{P}<0.05$ was considered to indicate a statistically significant difference. A total of 90 cases of $\mathrm{HCC}$ were included in the present study. AQP1 was markedly expressed in the membranes of microvessels and small vessels, but seldom in hepatocellularcarcinoma cells. Blood vessels in the tumors were markedly stained by anti-cluster of differentiation 34 antibody. AQP1 expression and IMD was significantly correlated with tumor size, histologic grade, Child-Pugh classification, microvascular invasion and tumor-node-metastasis (TNM) stage $(\mathrm{P}<0.05)$. Concurrently, for the 5-year DFS and OS, a larger tumor size, poorly differentiated histological grade, B and C Child-Pugh classification, presence of microvascular invasion, high TNM stage, a high AQP1 expression and a high IMD were significant risk factors for mortality. Multivariate analysis revealed that TNM stage and IMD were independent unfavorable prognostic markers for 5-year DFS $(\mathrm{P}=0.049$ and $\mathrm{P}=0.025$, respectively) and $\mathrm{OS}(\mathrm{P}=0.043$ and $\mathrm{P}=0.042$,
\end{abstract}

Correspondence to: Dr Min Wei, Clinical Laboratory, Nanshan Maternity \& Child Healthcare Hospital, 1 Wan Xia Road, Shenzhen, Guangdong 518067, P.R. China

E-mail: weiminphd@126.com

*Contributed equally

Key words: aquaporin-1, intratumoral microvessel density, immunohistochemistry, prognosis, hepatocellular carcinoma respectively). These data suggest that high AQP1 expression and IMD are associated with tumor progression and prognosis in HCC. The IMD level may serve as an independent indicator for the 5-year DFS and OS.

\section{Introduction}

It is well-known that hepatocellular carcinoma (HCC) is one of the most common types of malignancy in South-East Asia (1). For localized tumors, effective treatments include surgical resection, local ablation therapy, trans-catheter arterial chemoembolization and liver transplantation (1-3). However, $\mathrm{HCC}$ is typically diagnosed at the advanced stages in numerous patients. Although several molecular targeting drugs have previously been used in a clinical setting, their effects are limited (4). Therefore, novel molecular targets are required to manage HCC progression.

HCC is a typical hypervascular tumor, as demonstrated by dynamic computerized tomography or angiography $(5,6)$, and its progression is markedly associated with active neovascularization (7-9). Angiogenesis is important to tumor metastasis and growth, as it provides the oxygen, and nutrients for tumor cells $(10,11)$. Intratumoral microvessel density (IMD), the most common indicator of tumor angiogenesis, is assessed using cluster of differentiation (CD)31, CD34 or von Willebrand factor (vWF) staining (12). It has been suggested that an increased IMD is a predictor for decreased disease-free survival (DFS) and overall survival (OS) rates, and several antiangiogenic agents have begun to be used in the treatment of HCC $(9,13)$. However, conflicting results have also identified that a low IMD is a significant unfavorable prognostic factor of 2-year DFS as well as OS rate (14).

Aquaporins (AQPs) are a family of transmembrane water channel proteins, which are expressed in numerous types of fluid-transporting tissue, including glandular epithelia and kidney tubules, and in non-fluid-transporting tissue, including the epidermis. There are more than $10 \mathrm{AQPs}$ that have been identified in mammals (15). Their localization in the plasma membrane is essential in the regulation of water transfer (16). The first member to be identified, AQP1, is a membrane protein that regulates the permeability of endothelial and 
epithelial barriers by facilitating water movement across cell membranes (17). In addition to its basic function, human AQP1 expression has been revealed to be heterogeneously expressed in different human tumors (18-23). Several studies have identified that the upregulation of AQP1 occurs in various malignancies, including in glial tumors (18), breast cancer (19) and colorectal cancer (20). Furthermore, previous studies have investigated AQP1 expression in the microvessels of multiple tumors, indicating the potential involvement of AQP1 in tumor angiogenesis (17,21). Impaired tumor angiogenesis and tumor migration were identified in AQP1 knockout mice (22). Conversely, AQP1 overexpression is consistent with bone marrow angiogenesis in patients with active multiple myeloma, suggesting AQP1 is an indicator of angiogenesis (23).

However, the role of AQPs in HCC is poorly characterized. In the present study, the protein expression of AQP1 in HCC tissue samples was investigated, and the clinicopathological and prognostic value of AQP1 in HCC was analyzed.

\section{Materials and methods}

Tissue specimens and clinical data. Tumor samples and adjacent liver tissues were collected from 90 patients with HCC who underwent curative surgical resection without any prior anticancer therapy between May 2007 and May 2012 at the Centre for Liver Disease in the 458th Hospital of People's Liberation Army (Guangzhou, China). Patients with concurrent second primary cancer were excluded. The present study was approved by the Human Research Ethics Committee of the 458th Hospital of People's Liberation Army, and written informed consent was obtained from each patient. OS and DFS were defined as the interval between dates of surgery and mortality, and between dates of surgery and recurrence, respectively. Those patients who developed recurrence were treated with repeated hepatic resection, trans-catheter arterial embolization or radiofrequency ablation. Demographical and clinicopathological data consisted of age, sex, presence of cirrhosis, status of hepatitis B surface antigen (HBsAg), levels of preoperative $\alpha$-fetoprotein (AFP), tumor size, histological grade, Child-Pugh classification, microvascular invasion and tumor-node-metastasis (TNM) stage (14,24-26). Serial sections ( $5 \mu \mathrm{m}$ thick) were obtained from each tissue block, and stained with hematoxylin and eosin (H\&E; 0.2\% hematoxylin and $1 \%$ eosin) using standard pathologic procedures for $1 \mathrm{~h}$. Briefly, sections were deparaffinized in xylene (2x5 min) and rehydrated with successive 1-min washes in 100, 96, 80 and $70 \%$ ethanol. Sections were then stained with hematoxylin for $2 \mathrm{~min}$ at room temperature, rinsed with distilled water, rinsed with $0.1 \%$ hydrochloric acid in $50 \%$ ethanol, rinsed with tap water for $15 \mathrm{~min}$, stained with eosin for $1 \mathrm{~min}$ at room temperature and rinsed again with distilled water. The slides were then dehydrated with 95 and $100 \%$ ethanol successively followed by xylene $(2 \times 5 \mathrm{~min})$, and then mounted with coverslips. H\&E-stained sections were analyzed by light microscopy (magnification, x20) using a Leica DM LB2 epifluorescence microscope (Leica Microsystems GmbH, Wetzlar, Germany). Images that were at the original magnification of x 20 of $\mathrm{H} \& \mathrm{E}$ staining were acquired with a CCD digital camera (model 7.2; Diagnostic Instruments, Inc., Sterling Heights, MI, USA). The mean age of the patients was $54.0 \pm 10.0$ years (standard deviation; range, 25-73). There were 73 males and 17 females. The average tumor size was $4.4 \pm 1.8 \mathrm{~cm}$ (range, 1.3-7.7), with 47 tumors $\leq 5 \mathrm{~cm}$ and 43 tumors $>5 \mathrm{~cm}$. Among the $90 \mathrm{HCC}$ examined in the present study, 53 exhibited hepatitis B infection. The median follow-up time was 35.0 months.

Tissue microarray (TMA) construction and immunohistochemistry $(I H C)$. Immunohistochemistry images were captured and analyzed using Image Pro-Plus 4.5 software (Media Cybernetics, Silver Spring, MA, USA) for integrated optical density semi-quantitation. Leica DM LB2 epifluorescence microscope (Leica Microsystems $\mathrm{GmbH}$, Wetzlar, Germany) was used to analyze the images of IHC at a low magnification (x100) and a high magnification (x400). Representative sections of HCC or normal liver tissues in the pre-existing paraffin-embedded tissue blocks were determined according to the aforementioned H\&E staining slides. The TMA was prepared using a needle to punch a $1.5 \mathrm{~mm}$ diameter cylinder in the representative section of each tissue, and by placing the cylinders into an array on a recipient paraffin block. Sections were cut $2-\mu \mathrm{m}$ thick from the TMA block and mounted on microscope slides. The TMA consisted of a total of 90 patients with HCC and 90 cases of paraffin-embedded adjacent normal tissue. The clinicopathological characteristics of patients are summarized in Table I. The TMA slides were dried overnight at $37^{\circ} \mathrm{C}$, dewaxed in xylene, rehydrated using an alcohol gradient, and the endogenous peroxidase activity was blocked by immersing the slides in $0.3 \%$ hydrogen peroxide $\left(\mathrm{H}_{2} \mathrm{O}_{2}\right)$ for $10 \mathrm{~min}$ at room temperature. Antigen retrieval was performed through microwave heating with sodium citrate buffer $(\mathrm{pH} 6.0)$ at $100^{\circ} \mathrm{C}$ for $30 \mathrm{~min}$. Then, non-specific binding sites were blocked at room temperature using the blocking buffer from the Vectastain ${ }^{\circledR}$ Elite ABC kit (Vector Laboratories, Peterborough, UK) for $45 \mathrm{~min}$. Samples were then incubated with mouse monoclonal anti-human antibody against AQP1 (1:500 dilution; cat. no. ab9566; Abcam, Cambridge, MA, USA) and mouse monoclonal CD34 (1:50 dilution; cat. no. MA1-10202; clone QB End10; Neomarkers, Inc., Fremont, CA, USA) primary antibodies at room temperature for $60 \mathrm{~min}$. Following three washes with PBS, the slides were sequentially incubated with a polymer peroxidase-labeled rabbit anti-mouse secondary antibody (100 dilution; cat. no. ZDR-5109; ZSGB-BIO, Beijing, China) for $30 \mathrm{~min}$ at room temperature. Then, the slides were stained at $37^{\circ} \mathrm{C}$ for $1 \mathrm{~h}$ using the 3,3'-diaminobenzidine horseradish peroxidase Color Development kit (Beyotime Institute of Biotechnology, Haimen, China). Finally, the sections were counterstained with hematoxylin for $5 \mathrm{~min}$ at room temperature. Known IHC positive slides were used as a positive control, and anti-AQP1 primary antibody was replaced with PBS as a negative control.

Evaluation of IMD and AQP1 expression. IMD scores were assessed by immunostaining for CD34 according to Weidner (24). Subsequent to scanning the immunostained section at a low magnification (x100), the area within the tumor or adjacent tissues with the highest number of distinctly highlighted microvessels was selected as the 'hot spot'. IMD was defined by the mean value of vessel number visualized at high magnification (x400) in five fields within the hot spot. Evaluation of the staining reactions was strictly confined to 
Table I. Clinicopathological characteristics of patients with hepatocellular carcinoma.

\begin{tabular}{|c|c|}
\hline Characteristic & No. of cases $(\%$ \\
\hline \multicolumn{2}{|l|}{ Age, years } \\
\hline$\leq 60$ & $65(72.2)$ \\
\hline$>60$ & $25(27.8)$ \\
\hline \multicolumn{2}{|l|}{ Sex } \\
\hline Male & $73(81.1)$ \\
\hline Female & 17 (18.9) \\
\hline \multicolumn{2}{|l|}{ Cirrhosis } \\
\hline Absent & $59(65.6)$ \\
\hline Present & $31(34.4)$ \\
\hline \multicolumn{2}{|l|}{ Hepatitis B surface antigen } \\
\hline Negative & $36(40.0)$ \\
\hline Positive & $54(60.0)$ \\
\hline \multicolumn{2}{|l|}{$\alpha$-fetoprotein, $\mathrm{ng} / \mathrm{ml}$} \\
\hline$\leq 100$ & $32(35.6)$ \\
\hline$>100$ & $58(64.4)$ \\
\hline \multicolumn{2}{|l|}{ Tumor size, $\mathrm{cm}$} \\
\hline$\leq 5$ & $47(52.2)$ \\
\hline$>5$ & $43(47.8)$ \\
\hline \multicolumn{2}{|l|}{ Histological grade } \\
\hline Well differentiated & $25(27.8)$ \\
\hline Moderately differentiated & $55(61.1)$ \\
\hline Poorly differentiated & $10(11.1)$ \\
\hline \multicolumn{2}{|l|}{ Child-Pugh classification } \\
\hline A & $66(73.3)$ \\
\hline $\mathrm{B}-\mathrm{C}$ & $24(26.7)$ \\
\hline \multicolumn{2}{|l|}{ Microvascular invasion } \\
\hline Absent & $65(72.2)$ \\
\hline Present & $25(27.8)$ \\
\hline \multicolumn{2}{|l|}{ Tumor node metastasis stage } \\
\hline I-II & $50(55.6)$ \\
\hline III-IV & $40(44.4)$ \\
\hline
\end{tabular}

the area of highest IMD. For the sinusoid-like microvessels, which were primarily observed in the areas with a large trabecular structure and assessed using a modified method introduced by Tanigawa et al (27), every $40-\mu \mathrm{m}$ length of lumen was counted as 1 point. Each stained lumen was regarded as a single countable microvessel. If there was no lumen, but only a single positive cell was visible, this cell was also interpreted as representing a microvessel. Any positive staining of endothelium or mass of endothelium clearly separated from the surrounding tumor cells and connective tissue was counted as a microvessel. Immunohistochemical analysis was performed independently by two investigators (Dr Li-Min Luo, 458th Hospital of People's Liberation Army and Dr Min Wei, Southern Medical University). The mean values were accepted if the two investigators agreed with the values. If the differences between the observers were $>30 \%$, the values were re-estimated until a consensus was reached. The expression
A

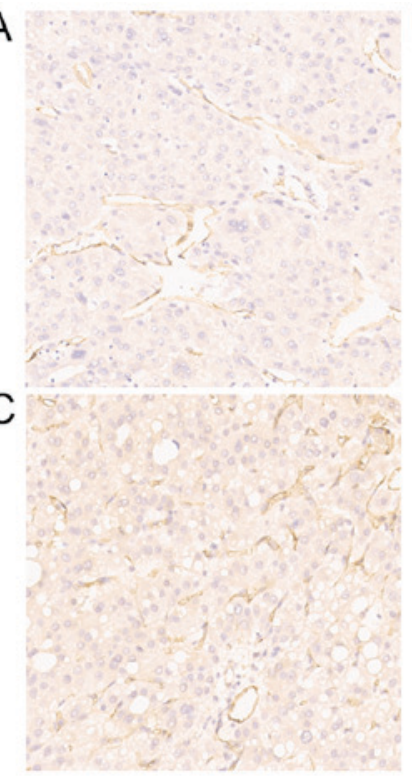

B

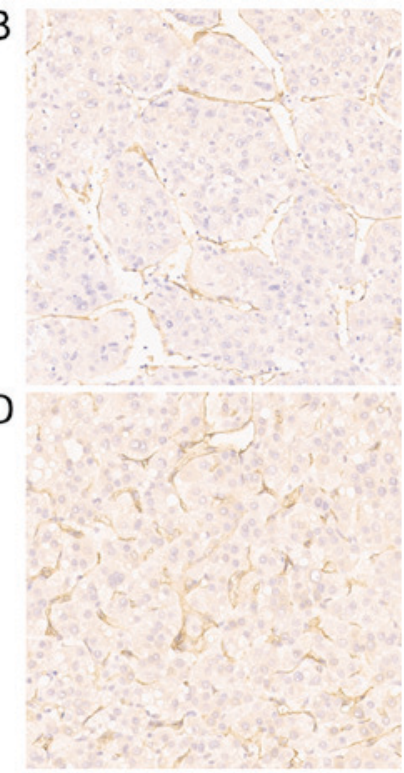

Figure 1. Representative images of immunohistochemical staining of hepatocellular carcinoma tissue samples. Immunohistochemical staining of capillary-like microvessels for (A) AQP1 and (B) CD34.Immunohistochemical staining of sinusoid-like microvessels for (C) AQP1 and (D) CD34. Magnification, x200. AQP1, aquaporin-1; CD, cluster of differentiation.

of AQP1 was detected and assessed using the same method. As the number of microvessels observed may vary by patient and vascular spots, resulting in an error in any measurement of AQP1 expression, the AQP1/IMD ratio was also assessed to avoid this error.

Statistical analysis. Statistical analysis was performed using SPSS software (version 18.0; SPSS, Inc., Chicago, USA). The associations between clinical and prognostic variables (patient age, sex, cirrhosis, HBsAg, AFP, tumor size, histological grade, Child-Pugh classification, microvascular invasion and TNM stage, and AQP1 expression, IMD and the AQP1/IMD ratio) were determined. Un-paired Student's t-tests were used to compare values between two groups, and one-way analysis of variance was performed when $\geq 3$ groups were present. Correlations were determined by Spearman rank correlation test. A two-tailed $\mathrm{P}<0.05$ was considered to indicate a statistically significant difference.

\section{Results}

Association between the AQP1 expression/IMD and the clinicopathological factors of the patients. There were two types of microvessels identified: Capillary-like microvessels with small, scattered capillaries with no or a narrow lumen, and sinusoid-like microvessels with continuous branching and a distinct lumen structure. Immunohistochemical analysis demonstrated that the AQP1 protein was markedly expressed in the membrane of microvessels and small vessels in the majority of HCC samples (Fig. 1A and B), but seldom in the cytoplasm of tumor cells. The AQP1 expression in microvessels of HCC presented a significant association with cirrhosis, tumor size, histological grade, Child-Pugh classification, microvascular invasion and TNM stage. The expression of 
Table II. Association between AQP1 expression, IMD, the AQP1/IMD ratio and the clinicopathological features of 90 patients with hepatocellular carcinoma.

\begin{tabular}{|c|c|c|c|c|c|c|c|}
\hline Features & Cases (n) & AQP1 & P-value & IMD & P-value & AQP1/IMD & P-value \\
\hline Cirrhosis & & & 0.048 & & 0.295 & & 0.051 \\
\hline Absent & 59 & $102.9 \pm 9.4$ & & $122.0 \pm 10.6$ & & $0.84 \pm 0.04$ & \\
\hline Present & 31 & $106.9 \pm 8.4$ & & $124.5 \pm 10.3$ & & $0.86 \pm 0.03$ & \\
\hline Hepatitis B surface antigen & & & 0.189 & & 0.265 & & 0.698 \\
\hline Negative & 36 & $105.8 \pm 9.9$ & & $124.4 \pm 10.8$ & & $0.85 \pm 0.04$ & \\
\hline Positive & 54 & $103.2 \pm 8.6$ & & $121.8 \pm 10.3$ & & $0.85 \pm 0.04$ & \\
\hline$\alpha$-fetoprotein, $\mathrm{ng} / \mathrm{ml}$ & & & 0.372 & & 0.677 & & 0.358 \\
\hline$\leq 25$ & 32 & $103.1 \pm 8.9$ & & $122.2 \pm 10.2$ & & $0.84 \pm 0.05$ & \\
\hline$>25$ & 58 & $104.9 \pm 9.4$ & & $123.2 \pm 10.7$ & & $0.85 \pm 0.03$ & \\
\hline Tumor size, $\mathrm{cm}$ & & & $<0.001$ & & $<0.001$ & & 0.259 \\
\hline$\leq 5$ & 47 & $100.2 \pm 6.3$ & & $118.7 \pm 7.7$ & & $0.85 \pm 0.04$ & \\
\hline$>5$ & 43 & $108.7 \pm 10.0$ & & $127.3 \pm 11.4$ & & $0.85 \pm 0.03$ & \\
\hline Histological grade & & & 0.001 & & 0.002 & & $<0.001$ \\
\hline Well differentiated & 25 & $100.8 \pm 7.2$ & & $119.3 \pm 8.3$ & & $0.85 \pm 0.04$ & \\
\hline Moderately differentiated & 55 & $104.1 \pm 9.1$ & & $122.7 \pm 10.7$ & & $0.85 \pm 0.04$ & \\
\hline Poorly differentiated & 10 & $113.7 \pm 8.3$ & & $132.7 \pm 8.9$ & & $0.86 \pm 0.02$ & \\
\hline Child-Pugh classification & & & 0.007 & & 0.019 & & 0.366 \\
\hline A & 66 & $102.7 \pm 8.8$ & & $121.3 \pm 9.6$ & & $0.85 \pm 0.04$ & \\
\hline $\mathrm{B}-\mathrm{C}$ & 24 & $108.6 \pm 9.2$ & & $127.1 \pm 11.8$ & & $0.86 \pm 0.03$ & \\
\hline Microvascular invasion & & & $<0.001$ & & $<0.001$ & & 0.506 \\
\hline Absent & 65 & $101.2 \pm 7.0$ & & $110.0 \pm 8.3$ & & $0.85 \pm 0.04$ & \\
\hline Present & 25 & $112.3 \pm 9.5$ & & $132.9 \pm 9.0$ & & $0.84 \pm 0.04$ & \\
\hline Tumor node metastasis stage & & & $<0.001$ & & $<0.001$ & & 0.278 \\
\hline I-II & 50 & $99.7 \pm 5.5$ & & $118.0 \pm 7.0$ & & $0.84 \pm 0.04$ & \\
\hline III-IV & 40 & $110.0 \pm 9.7$ & & $128.9 \pm 10.1$ & & $0.85 \pm 0.03$ & \\
\hline
\end{tabular}

Data are presented as the mean \pm standard deviation, Student's t-test; $\mathrm{P}<0.05$ were considered to indicate a statistically significant difference. AQP1, aquaporin-1; IMD, intratumoral microvessel density.

AQP1 was significantly higher in the presence of cirrhosis compared with in absence of cirrhosis $(\mathrm{P}=0.048)$, in tumor sizes $>5 \mathrm{~cm}$ compared with in tumor sizes $\leq 5 \mathrm{~cm}(\mathrm{P}<0.001)$, in poorly differentiated histological grades compared with in well or moderately differentiated histological grade $(\mathrm{P}=0.001)$, in Child-Pugh classification B + C compared with in Child-Pugh classification $\mathrm{A}(\mathrm{P}=0.007)$, in the presence of microvascular invasion compared with in absence of microvascular invasion $(\mathrm{P}<0.001)$ and in TNM stage III-IV compared with in TNM stage I-II $(\mathrm{P}<0.001)$ (Table II). However, no significant variations according to HBsAg and AFP levels were observed ( $\mathrm{P}>0.05$; Table II). CD34 was also highly expressed in the membrane of microvessels and small vessels in the majority of HCC samples (Fig. 1C and D). The IMD score, assessed by CD34 immunostaining, was significantly associated with tumor size, histological grade, Child-Pugh classification, microvascular invasion and TNM stage. IMD scores were higher in tumor sizes $>5 \mathrm{~cm}$ compared with in tumor sizes $\leq 5 \mathrm{~cm}(\mathrm{P}<0.001)$, in poorly differentiated histological grade compared with in well and moderately differentiated histological grades $(\mathrm{P}=0.002)$, in Child-Pugh classification B + C compared with in Child-Pugh classification A $(\mathrm{P}=0.019)$, in the presence of microvascular invasion compared with in absence of microvascular invasion $(\mathrm{P}<0.001)$ and in TNM stage III-IV compared with in TNM stage I-II $(\mathrm{P}<0.001)$ (Table II). However, no significant differences between IMD score and cirrhosis, HBsAg or AFP were observed ( $\mathrm{P}>0.05$; Table II). A statistically significant positive correlation was observed between AQP1 expression and the IMD scores $(\mathrm{r}=0.227 ; \mathrm{P}<0.001)$.

Analysis of the AQP1/IMD ratio. The AQP1/IMD ratio in cases with poorly differentiated histological grade was significantly greater compared with that of cases with well and moderately differentiated histological grades $(\mathrm{P}<0.001)$. However, no significant differences between AQP1/IMD ratio and cirrhosis, HBsAg, AFP, tumor size, Child-Pugh classification, microvascular invasion and TNM stage were observed (P>0.05; Table II).

Prognostic value of AQP1 expression or IMD on overall survival and recurrence. Univariate analysis of factors revealed that tumor size, histological grade, Child-Pugh 
Table III. Univariate and multivariate analysis of factors associated with survival, and recurrence.

\begin{tabular}{|c|c|c|c|c|c|c|c|c|}
\hline \multirow[b]{3}{*}{ Factor } & \multicolumn{4}{|c|}{ Disease free survival } & \multicolumn{4}{|c|}{ Overall survival } \\
\hline & \multirow{2}{*}{$\begin{array}{l}\text { Univariate } \\
\text { P-value }\end{array}$} & \multicolumn{3}{|c|}{ Multivariate } & \multirow{2}{*}{$\begin{array}{l}\text { Univariate } \\
\text { P-value }\end{array}$} & \multicolumn{3}{|c|}{ Multivariate } \\
\hline & & HR & $95 \% \mathrm{CI}$ & P-value & & HR & $95 \% \mathrm{CI}$ & P-value \\
\hline Cirrhosis (absent vs. present) & 0.071 & 0.911 & $0.393-2.112$ & 0.828 & 0.062 & 0.811 & $0.334-1.968$ & 0.643 \\
\hline $\begin{array}{l}\text { Hepatitis B surface antigen } \\
\text { (negative vs. positive) }\end{array}$ & 0.744 & 1.924 & $0.887-4.175$ & 0.098 & 0.808 & 1.822 & $0.841-3.949$ & 0.129 \\
\hline$\alpha$-fetoprotein $(\leq 25 \mathrm{vs} .>25 \mathrm{ng} / \mathrm{ml})$ & 0.347 & 1.248 & $0.567-2.749$ & 0.582 & 0.251 & 1.443 & $0.632-3.296$ & 0.384 \\
\hline Tumor size ( $\leq 5$ vs. $>5 \mathrm{~cm})$ & $<0.001$ & 1.265 & $0.484-3.307$ & 0.631 & $<0.001$ & 1.316 & $0.511-3.389$ & 0.570 \\
\hline $\begin{array}{l}\text { Histological grade } \\
\text { (well or moderately vs. poorly) }\end{array}$ & $<0.001$ & 1.487 & $0.579-3.819$ & 0.409 & $<0.001$ & 1.656 & $0.625-4.385$ & 0.310 \\
\hline Child-Pugh (A vs. B-C) & 0.035 & 1.010 & $0.404-2.526$ & 0.983 & 0.018 & 1.084 & $0.418-2.813$ & 0.868 \\
\hline $\begin{array}{l}\text { Microvascular invasion } \\
\text { (absent vs. present) }\end{array}$ & $<0.001$ & 1.638 & $0.766-3.502$ & 0.203 & $<0.001$ & 1.726 & $0.814-3.660$ & 0.155 \\
\hline $\begin{array}{l}\text { Tumor node metastasis stage } \\
\text { (I-II vs. III-IV) }\end{array}$ & $<0.001$ & 2.867 & $1.005-3.502$ & 0.049 & $<0.001$ & 2.921 & $1.033-8.261$ & 0.043 \\
\hline AQP1 expression (low vs. high) & 0.001 & 0.636 & $0.228-1.772$ & 0.387 & $<0.001$ & 0.770 & $0.279-2.127$ & 0.615 \\
\hline IMD (low vs. high) & $<0.001$ & 3.444 & $1.169-10.149$ & 0.025 & $<0.001$ & 3.074 & $1.039-9.094$ & 0.042 \\
\hline AQP1/IMD ratio (low vs. high) & 0.753 & 0.959 & $0.489-1.880$ & 0.903 & 0.899 & 0.999 & $0.502-1.988$ & 0.998 \\
\hline
\end{tabular}

$\mathrm{P}<0.05$ were considered to indicate a statistically significant difference. HR, hazard ratio; CI, confidence interval; AQP1, aquaporin-1; IMD, intratumoral microvessel density.

classification, microvascular invasion, TNM stage, AQP1 expression and IMD were associated with 5-year DFS, and OS (Table III). The median 5-year DFS and OS times of patients with low AQP1 expression were significantly longer compared with that of patients with high AQP1 expression $(\mathrm{P}=0.001$ for both; Fig. 2A and B; Table III). Similarly, patients with a high IMD exhibited significantly shorter 5-year DFS and OS times ( $\mathrm{P}<0.001$ for both; Fig. 2C and D; Table III). Low AQP1 expression and low IMD were independent protective factors of 5-year DFS ( $\mathrm{P}=0.001$ and $\mathrm{P}<0.001$, respectively) and $\mathrm{OS}$ $(\mathrm{P}<0.001$ and $\mathrm{P}<0.001$, respectively) (Table III). Notably, in the multivariate analyses, IMD biomarker was an independent risk factor of 5-year OS ( $\mathrm{P}=0.042$; Fig. 2C) and DFS ( $\mathrm{P}=0.025$; Fig. 2D) (Table III). In addition, TNM stage was identified as an independent risk factor for $\mathrm{OS}(\mathrm{P}=0.043$; Fig. 2E) and DFS ( $\mathrm{P}=0.049$; Fig. 2F) (Table III).

\section{Discussion}

The ability of tumor cells to grow and migrate requires a sufficient blood supply. A number of malignant tumors have been identified to induce neovascularization $(28,29)$. Tanigawa et al (27) demonstrated an increased microvessel density in malignant HCC and indicated that IMD was a prognostic factor for HCC. However, the clinicopathological significance of angiogenesis in HCC remains to be elucidated $(13,14,30)$. Due to the diversities in tissue processing and immunostaining techniques, including the observation for selected vascular hot spots, antibodies to identify endothelial cells, and the method of counting the vessels, the results of angiogenesis are not able to be corroborated easily.
Anti-CD34 antibodies have been identified to be better at identifying endothelial cells compared with anti-CD31 and anti-vWF antibodies, and with greater sensitivity $(13,14,27)$. The anti-CD34 antibody has been suggested to be the most sensitive and specific marker among the other endothelial markers in HCC (31). Therefore, IMD score was assessed using the anti-CD34 antibody in the present study. The results suggest that IMD may serve an important role in the HCC due to its association with tumor size, histological grade, Child-Pugh classification, microvascular invasion and TNM stage, which was in accordance with Tanigawa et al (27), and Wang et al (32), who hypothesized that IMD is an independent prognostic factor for HCC.

The number of microvessels in tumors varies in different patients or hot spots, which may result in differences in AQP1 expression measurements. For IMD, defined as tumor microvessel counts, and AQP1 protein, which is primarily expressed in microvessels, the AQP1/IMD ratio may determine the association between IMD and AQP1 expression levels in the microvessels of HCC, and correct subjective and objective errors. In the present study, AQP1 protein was highly expressed in the membranes of microvessels and small vessels within the majority of patients with HCC, but was expressed seldom in the cytoplasm of the tumor cells. The distribution of AQP1 protein indicated that AQP1 may serve an important role in transvascular water transport in primary $\mathrm{HCC}$, and exhibits little effect on water flow in tumor cells.

The expression of AQP1 in HCC tissues was higher compared with that of adjacent normal liver tissues. These data indicate the potential role of AQP1 during HCC carcinogenesis. It is possible that the induction of AQP1 is required in the development of 
A

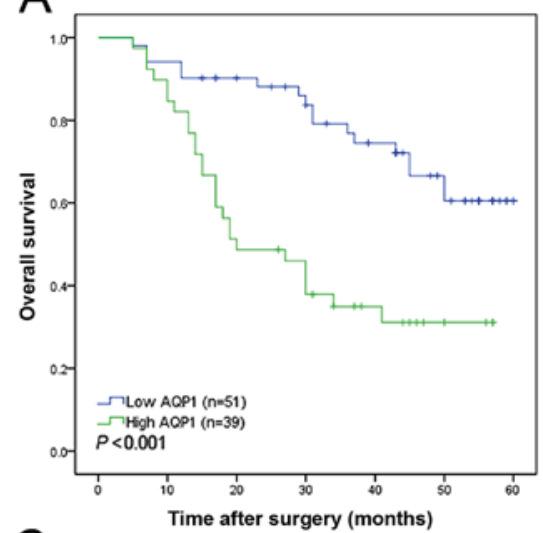

C

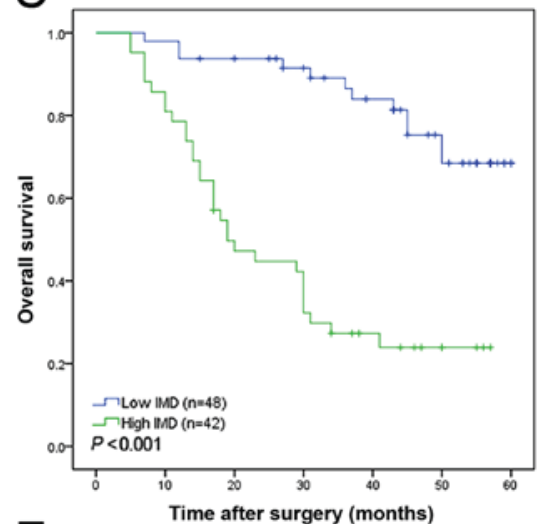

E

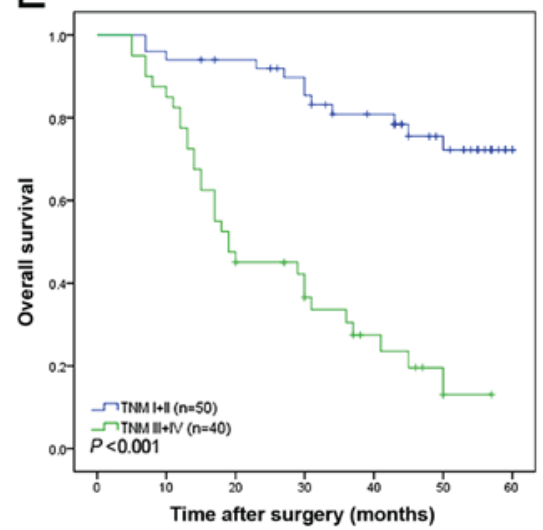

B

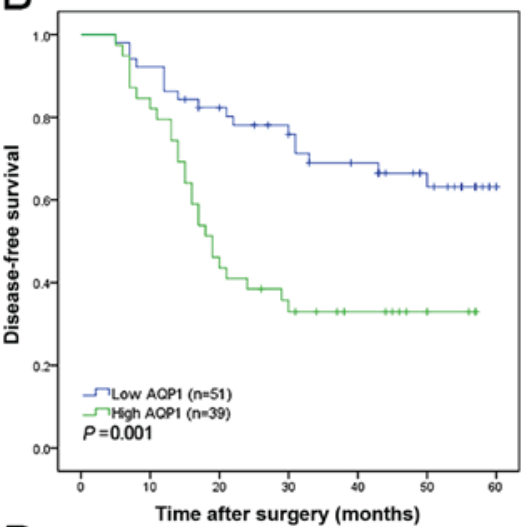

D

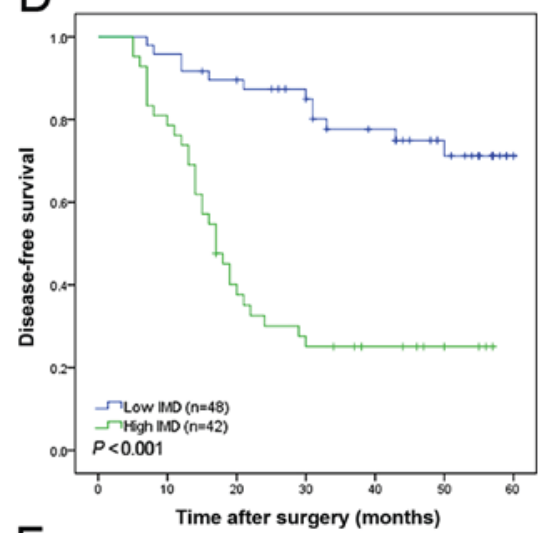

$\mathrm{F}$

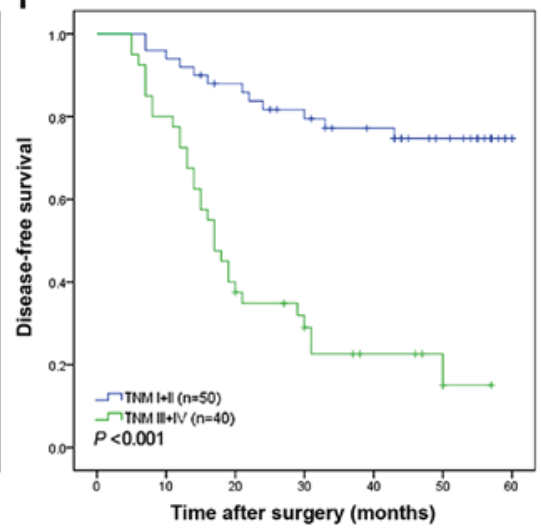

Figure 2. Cumulative OS and DFS curves of 90 patients with hepatocellular carcinoma. (A) OS curves of patients classified by AQP1 expression. (B) DFS curves of patients classified by AQP1 expression. (C) OS curves of patients classified by IMD score. (D) DFS curves of patients classified by IMD score. (E) OS curves of patients classified by TNM stage. (F) DFS curves of patients classified by TNM stage. OS, overall survival; DFS, disease-free survival; AQP1, aquaporin-1; TNM, tumor node metastasis.

$\mathrm{HCC}$ and serves as an essential driving force for initiating carcinogenesis. During the cell cycle, as the cell volume needs to expand rapidly by absorbing water from the extracellular environment with a minimal volume of energy, upregulation of AQP1 in microvessels is potentially advantageous for the growth or survival of tumor cells (33). Furthermore, the result suggests that HCC, similar to other solid tumors, exhibit high vascular permeability (34).

Previous studies have demonstrated that AQP1 expression is upregulated in astrocytomas and metastatic carcinomas $(35,36)$, and AQP1 expression in the microvessels of neoplastic brain cells was proposed to increase blood-brain barrier water permeability, resulting in brain tumor edema in aggressive brain tumors (37).
In addition, the results of the present study indicated that AQP1 expression in the microvessels of HCC samples was significantly associated with tumor size, histologic grade, Child-Pugh classification, microvascular invasion and TNM stage. The survival analysis results suggested that the AQP1 protein may be upregulated in the advanced stages of the disease, and may be involved in the progression and prognosis of HCC.

In the present study, Spearman correlations demonstrated that there was a positive correlation between IMD and the expression of $\mathrm{AQP} 1$. These results suggest that $\mathrm{AQP} 1$ expression in microvessel endothelial cells of HCC may be associated with angiogenesis. Additional experiments are required to investigate whether AQP1 overexpression or knockout in tumor microvessels affect angiogenesis directly. 
Papadopoulos and Verkman (38) demonstrated that the pharmacological modulation of AQP1 function may provide novel therapeutic approaches in human disease, including diuretics, and regulators of intraocular pressure and swelling in the brain, and cornea. In addition, Ma et al (39) suggested that topiramate decreases AQP1 protein immunostaining in lung carcinoma microvessel endothelial cells of mice, and hypothesized that the suppression of AQP1 expression may be an important factor for the inhibitory action of topiramate on tumor metastasis. In conclusion, the results of the present study indicate that high AQP1 expression may serve an essential role in HCC carcinogenesis and progression. Additional studies investigating the molecular mechanisms of AQP1 regulation, and the association between AQP1 expression and tumor angiogenesis, are required to verify this novel therapy for HCC.

\section{Acknowledgements}

The present study was supported by the National Natural Science Foundation of China (grant no. 81672754), the Natural Science Foundation of Guangdong province (grant no. 2015A030313249) and the 'Twelfth Five Year Plan' research project, Medical Department of General Logistics Department of China (grant no. CWS11J021).

\section{References}

1. Llovet JM: Updated treatment approach to hepatocellular carcinoma. J Gastroenterology 40: 225-235, 2005.

2. Bruix J, Sala M and Llovet JM: Chemoembolization for hepatocellular carcinoma. Gastroenterology 127 (5 Suppl 1): S179-S188, 2004.

3. Gish RG, Marrero JA and Benson AB: A multidisciplinary approach to the management of hepatocellular carcinoma. Gastroenterol Hepatol (NY) 6 (3 Suppl 6): 1S-16S, 2010.

4. Llovet JM, Ricci S, Mazzaferro V, Hilgard P, Gane E, Blanc JF, de Oliveira AC, Santoro A, Raoul JL, Forner A, et al: Sorafenib in advanced hepatocellular carcinoma. N Engl J Med 359: 378-390, 2008

5. Gomaa AI, Khan SA, Leen EL, Waked I and Taylor-Robinson SD: Diagnosis of hepatocellular carcinoma. World J Gastroenterol 15: 1301-1314, 2009.

6. Ayyappan AP and Jhaveri KS: CT and MRI of hepatocellular carcinoma: An update. Exp Rev Anticancer Ther 10: 507-519, 2010.

7. Park YN, Kim YB, Yang KM and Park C: Increased expression of vascular endothelial growth factor and angiogenesis in the early stage of multistep hepatocarcinogenesis. Arch Pathol Lab Med 124: 1061-1065, 2000

8. Roncalli M, Roz E, Coggi G, Di Rocco MG, Bossi P, Minola E, Gambacorta $\mathrm{M}$ and Borzio $\mathrm{M}$ : The vascular profile of regenerative and dysplastic nodules of the cirrhotic liver: Implications for diagnosis and classification. Hepatology 30: 1174-1178, 1999.

9. Poon RT, Ng IO, Lau C, Zhu LX, Yu WC, Lo CM, Fan ST and Wong J: Serum vascular endothelial growth factor predicts venous invasion in hepatocellular carcinoma: A prospective study. Ann Surg 233: 227-235, 2001

10. Carmeliet $P$ and Jain RK: Angiogenesis in cancer and other diseases. Nature 407: 249-257, 2000.

11. Yao DF, Wu XH, Zhu Y, Shi GS, Dong ZZ, Yao DB, Wu W, Qiu LW and Meng XY: Quantitative analysis of vascular endothelial growth factor, microvascular density and their clinicopathologic features in human hepatocellular carcinoma. Hepatobiliary Pancreat Dis Int 4: 220-226, 2005.

12. Weidner N, Folkman J, Pozza F, Bevilacqua P, Allred EN, Moore DH, Meli S and Gasparini G: Tumor angiogenesis: A new significant and independent prognostic indicator in early-stage breast carcinoma. J Natl Cancer Inst 84: 1875-1887, 1992.

13. Sun HC, Tang ZY, Li XM, Zhou YN, Sun BR and Ma ZC: Microvessel density of hepatocellular carcinoma: Its relationship with prognosis. J Cancer Res Clin Oncol 125: 419-426, 1999.
14. Murakami K, Kasajima A, Kawagishi N, Ohuchi N and Sasano H: Microvessel density in hepatocellular carcinoma: Prognostic significance and review of the previous published work. Hepatol Res 45: 1185-1194, 2015.

15. Zhu C, Jiang Z, Bazer FW, Johnson GA, Burghardt RC and Wu G: Aquaporins in the female reproductive system of mammals. Front Biosci (Landmark Ed) 1: 838-871, 2015.

16. Saadoun S, Papadopoulos MC, Davies DC, Krishna S and Bell BA: Aquaporin-4 expression is increased in oedematous human brain tumours. J Neurol Neurosurg Psychiatry 72: 262-265, 2002.

17. Mobasheri A, Airley R, Hewitt SM and Marples D: Heterogeneous expression of the aquaporin 1 (AQP1) water channel in tumors of the prostate, breast, ovary, colon and lung: A study using high density multiple human tumor tissue microarrays. Int J Oncol 26: 1149-1158, 2005.

18. Oshio K, Binder DK, Liang Y, Bollen A, Feuerstein B, Berger MS and Manley GT: Expression of the aquaporin-1 water channel in human glial tumors. Neurosurgery 56: 375-381, 2005.

19. Mobasheri A and Barrett-Jolley R: Aquaporin water channels in the mammary gland: From physiology to pathophysiology and neoplasia. J Mammary Gland Biol Neoplasia 19: 91-102, 2014.

20. Yoshida T, Hojo S, Sekine S, Sawada S, Okumura T, Nagata T, Shimada Y and Tsukada K: Expression of aquaporin-1 is a poor prognostic factor for stage II and III colon cancer. Mol Clin Oncol 1: 953-958, 2013.

21. Endo M, Jain RK, Witwer B and Brown D: Water channel (aquaporin 1) expression and distribution in mammary carcinomas and glioblastomas. Microvascular Research 58: 89-98, 1999.

22. Saadoun S, Papadopoulos MC, Hara-Chikuma M and Verkman AS: Impairment of angiogenesis and cell migration by targeted aquaporin-1 gene disruption. Nature 434: 786-792, 2005.

23. Vacca A, Frigeri A, Ribatti D, Nicchia GP, Nico B, Ria R, Svelto M and Dammacco F: Microvessel overexpression of aquaporin 1 parallels bone marrow angiogenesis in patients with active multiple myeloma. Br J Haematol 113: 415-421, 2001.

24. Weidner N: Current pathologic methods for measuring intratumoral microvessel density within breast carcinoma and other solid tumors. Breast Cancer Res Treat 36: 169-180, 1995.

25. Zhang C, Bai DS, Huang XY, Shi GM, Ke AW, Yang LX, Yang XR, Zhou J and Fan J: Prognostic Significance of Capn4 overexpression in intrahepatic cholangiocarcinoma. PLoS One 8: e54619, 2013.

26. Kiriyama S, Uchiyama K, Ueno M, Ozawa S, Hayami S, Tani M and Yamaue H: Triple positive tumor markers for hepatocellular carcinoma are useful predictors of poor survival. Ann Surg 254: 984-991, 2011.

27. Tanigawa N, Lu C, Mitsui T and Miura S: Quantitation of sinusoid-like vessels in hepatocellular carcinoma: Its clinical and prognostic significance. Hepatology 26: 1216-1223, 1997.

28. Folkman J, Watson K, Ingber D and Hanahan D: Induction of angiogenesis during the transition from hyperplasia to neoplasia. Nature 339: 58-61, 1989.

29. Liotta LA and Stetler-Stevenson WG: Tumor invasion and metastasis: An imbalance of positive and negative regulation. Cancer Res 51 (18 Suppl): 5054s-5059s, 1991.

30. Chen ZY, Wei W, Guo ZX, Lin JR, Shi M and Guo RP: Morphologic classification of microvessels in hepatocellular carcinoma is associated with the prognosis after resection. J Gastroenterol Hepatol 26: 866-874, 2011.

31. Messerini L, Novelli L and Comin CE: Microvessel density and clinicopathological characteristics in hepatitis $\mathrm{C}$ virus and hepatitis B virus related hepatocellular carcinoma. J Clin Pathol 57: 867-871, 2004

32. Wang WQ, Liu L, Xu HX, Luo GP, Chen T, Wu CT, Xu YF, $\mathrm{Xu}$ J, Liu C, Zhang B, et al: Intratumoral $\alpha$-SMA enhances the prognostic potency of CD34 associated with maintenance of microvessel integrity in hepatocellular carcinoma and pancreatic cancer. PLoS One 8: e71189, 2013.

33. Moon C, Soria JC, Jang SJ, Lee J, Obaidul Hoque M, Sibony M, Trink B, Chang YS, Sidransky D and Mao L: Involvement of aquaporins in colorectal carcinogenesis. Oncogene 22: 6699-6703, 2003.

34. Yuan F, Leunig M, Huang SK, Berk DA, Papahadjopoulos D and Jain RK: Microvascular permeability and interstitial penetration of sterically stabilized (stealth) liposomes in a human tumor xenograft. Cancer Res 54: 3352-3356, 1994.

35. Saadoun S, Papadopoulos MC, Davies DC, Bell BA and Krishna S: Increased aquaporin 1 water channel expression in human brain tumours. Br J Cancer 87: 621-623, 2002. 
36. El Hindy N, Bankfalvi A, Herring A, Adamzik M, Lambertz N, Zhu Y, Siffert W, Sure U and Sandalcioglu IE: Correlation of aquaporin-1 water channel protein expression with tumor angiogenesis in human astrocytoma. Anticancer Res 33: 609-613, 2013.

37. Qin F, Zhang H, Shao Y, Liu X, Yang L, Huang Y, Fu L, Gu F and Ma Y: Expression of aquaporin1, a water channel protein, in cytoplasm is negatively correlated with prognosis of breast cancer patients. Oncotarget 16: 8143-8154, 2016.
38. Papadopoulos MC and Verkman AS: Potential utility of aquaporin modulators for therapy of brain disorders. Prog Brain Res 170: 589-601, 2008

39. Ma B, Xiang Y, Li T, Yu HM and Li XJ: Inhibitory effect of topiramate on Lewis lung carcinoma metastasis and its relation with AQP1 water channel. Acta Pharmacol Sin 25: 54-60, 2004. 